\title{
Structure of the $\mathrm{p} 53$ transactivation domain in complex with the nuclear coactivator binding domain of $\mathrm{CBP} \dagger$
}

\author{
Chul Won Lee, Maria A. Martinez-Yamout, H. Jane Dyson, and Peter E. Wright ${ }^{\star}$
}

\section{Abstract}

The activity and stability of the tumor suppressor p53 is regulated by interactions with key cellular proteins such as MDM2 and CBP/p300. The transactivation domain (TAD) of p53 contains two subdomains (AD1 and AD2) and interacts directly with the N-terminal domain of MDM2 and with several domains of CBP/p300. Here we report the NMR structure of the full-length p53 TAD in complex with the nuclear coactivator binding domain (NCBD) of CBP. Both the p53 TAD and NCBD are intrinsically disordered and fold synergistically upon binding, as evidenced by the observed increase in helicity and increased dispersion of the amide proton resonances. The p53 TAD folds to form a pair of helices (denoted $\mathrm{P} \alpha 1$ and $\mathrm{P} \alpha 2$ ), which extend from Phe19 to Leu25 and Pro47 to Trp53, respectively. In the complex, the NCBD forms a bundle of three helices (Ca1: residues 2066-2075, C $\alpha 2$ : residues 2081-2092, and Ca3: residues 2095-2105) with a hydrophobic groove into which the $\mathrm{p} 53$ helices $\mathrm{P} \alpha 1$ and $\mathrm{P} \alpha 2$ dock. The polypeptide chain between the p53 helices remains flexible and makes no detectable intermolecular contacts with the NCBD. Complex formation is driven largely by hydrophobic contacts that form a stable intermolecular hydrophobic core. A salt bridge between D49 of p53 and R2105 of NCBD may contribute to the binding specificity. The structure provides the first insights into simultaneous binding of the AD1 and $\mathrm{AD} 2$ motifs to a target protein.

The p53 tumor suppressor acts as a hub in signal transduction networks that mediate the cellular response to stress, leading to cell-cycle arrest, senescence, or apoptosis $(1,2)$. Due to its role in determining cell fate, $\mathrm{p} 53$ is tightly controlled by numerous regulatory proteins that include MDM2, MDMX, CBP/p300, and various kinases. In unstressed cells, p53 is maintained at extremely low levels through interactions with the ubiquitin E3 ligase MDM2 $(3,4)$. This interaction results in ubiquitination and proteasomal degradation of p53 and also blocks interactions with the basal transcriptional proteins $(5,6)$. MDMX, which is highly homologous to MDM2 but lacks ubiquitin ligase activity, also negatively regulates p53 and inhibits its transactivation function $(7,8)$. Upon cellular stress, specific kinases are activated which phosphorylate the $\mathrm{N}$-terminal region of $\mathrm{p} 53$. Phosphorylation facilitates release from MDM2 and enhances binding to the general transcriptional coactivators CBP and p300 (9$13)$.

CBP and p300 function as scaffolds for the recruitment and assembly of the transcriptional machinery and modify both chromatin and transcription factors through their intrinsic

\footnotetext{
$\dagger$ This work was supported by grant CA96865 from the National Institutes of Health. C.W.L. was supported by the Korea Research Foundation Grant funded by the Korean Government (MOEHRD, Basic Research Promotion Fund) (KRF-2004-214-C00207). *Corresponding author: Department of Molecular Biology and The Skaggs Institute for Chemical Biology, The Scripps Research Institute, 10550 North. Torrey Pines Road. La Jolla, California 92037, phone: 858784 9721, fax: 858784 9822, wright@ scripps.edu. Supporting Information Available

A schematic diagram showing the domains of CBP and p53 with their abbreviations (Figure S1), CD spectra of the free proteins and the complexes (Figure S2), graphs of $\mathrm{C} \alpha$ secondary chemical shifts for each component of the complex (Figure S3) and a graph showing the locations of paramagnetic relaxation enhancement to spin relaxation for p53(13-61) (Figure S4). This material is available free of charge via the Internet at http://pubs.acs.org.
} 
acetyltransferase activity (14). They contain multiple protein interaction domains, including TAZ1, KIX, TAZ2, and a disordered C-terminal domain known variously as the nuclear receptor coactivator binding domain (NCBD) (15), the IRF-3 binding domain (IBiD) (16), and the SRC1 interaction domain (SID) (17) (shown schematically in Supplementary Figure $\mathrm{S} 1 \mathrm{~A})$. CBP and p300 play a central role in the $\mathrm{p} 53$ response, and are required both for activation of p53-mediated transcription and for stabilization of the p53-DNA complex by acetylation of lysine residues in the C-terminal regulatory domain. Acetylation is necessary to inhibit the p53-MDM2 interaction and facilitate p53-mediated stress response (18).

p53 is a modular protein that binds DNA as a tetramer; each subunit is comprised of an Nterminal transactivation domain (TAD, residues 1-61), a proline-rich domain (PRD), a core DNA binding domain, the tetramerization domain, and a C-terminal regulatory domain (Figure S1B). The p53 TAD interacts with CBP/p300 at multiple sites, and binding to one or more of the TAZ1, TAZ2, KIX, and NCBD domains is required for CBP/p300-mediated transcription (19-26). The p53 TAD contains two subdomains, AD1 (residues 1-42) and AD2 (residues 43-63) (27-30) and is intrinsically disordered (28, 31, 32). NMR studies revealed that the free p53 TAD has a weak propensity to form transient helical structure between residues 18 and 26 and two turns (residues 40-44 and 48-53) in solution $(33,34)$. Regions within both AD1 and AD2 form stable helical structure upon binding to target proteins. Residues 18-26 in AD1 fold into an amphipathic helix upon binding to MDM2 (35), and helical structure is formed in AD2 upon binding to replication protein A and the $\mathrm{Tfb} 1$ subunit of TFIIH $(36,37)$.

We showed previously that the AD1 and AD2 activation domains within the p53 TAD mediate interactions with CBP and HDM2 (38). Binding to CBP is dominated by interactions with $\mathrm{AD} 2$, while binding to HDM2 is mediated primarily by interactions involving the AD1. The p53 TAD can bind simultaneously to HDM2 and to any one of CBP domains, through the AD1 and AD2 motifs, respectively, to form a ternary complex (39).

In spite of the importance of the $\mathrm{p} 53-\mathrm{CBP} / \mathrm{p} 300$ interaction, there is only limited structural information available, for the complex between the AD1 region of the p53 TAD and the TAZ2 domain of p300 (40). To provide further insights into the structural basis for CBP/ p300 recruitment by p53, we have determined the solution structures of the complex formed between the NCBD domain of CBP and residues 13-61 of the p53 TAD, encompassing both the AD1 and AD2 activation motifs. The NCBD is a key p53 binding site that contributes to activation of transcription of p53-responsive genes $(26,41)$. The NCBD has been identified as the binding site for diverse proteins with little sequence similarity, including adenoviral E1A, the interferon regulatory factor IRF-3, the p160 nuclear receptor coactivators ACTR, SRC1, and TIF-2, the human T-cell leukemia virus protein Tax, and p53, and undergoes large conformational changes upon binding to different protein targets $(16,42,43)$. Upon binding to the NCBD, the AD1 and AD2 regions of the disordered p53 TAD fold to form helices which are stabilized by docking in a hydrophobic groove on the surface of the NCBD. The loop between AD1 and AD2 remains flexible and wraps around the third helix of the NCBD. Binding occurs primarily through hydrophobic interactions, and a salt bridge between D49 of p53 and R2105 of the NCBD may contribute to the specificity.

\section{EXPERIMENTAL PROCEDURES}

\section{Protein Expression and Purification}

The NCBD (residues 2059-2117) domain of mouse CBP, and constructs from the TAD of human p53 [p53(1-61), p53(13-61), and p53(38-61)] were expressed and purified as described previously $(38,42)$. p53 $(25-61)$ was purified from protease digestion of p53(1361) using trypsin (Sigma). The peptide p53(14-28) was synthesized on a Perseptive 
Biosystems synthesizer using solid-phase Fmoc methods as described previously (38). p53(13-61)D49A was engineered using site-directed mutagenesis, and expressed and purified as described for the wild-type protein. For the NMR experiments, ${ }^{15} \mathrm{~N}-$ or ${ }^{13} \mathrm{C},{ }^{15} \mathrm{~N}$ labeled p53(13-61) and NCBD domains were expressed in Escherichia coli BL21(DE3) [DNAY] in M9 minimal medium and purified. Spin labeled $\left[{ }^{15} \mathrm{~N}\right] \mathrm{p} 53(13-61)$ was prepared by coupling MTSL to an N-terminal Cys residue, as described previously (44).

\section{Isothermal Titration Calorimetry (ITC)}

Binding interactions were measured by isothermal titration calorimetry using a MicroCal Omega VP-ITC instrument (MicroCal, Amherst, MA), as previously described (38).

\section{NMR Sample Preparation}

For NMR experiments, p53(13-61) and NCBD were dissolved in 10mM sodium phosphate, $\mathrm{pH} 6.5,50 \mathrm{mM} \mathrm{NaCl}$. The solution of the complex was dialyzed in the same buffer. The dialyzed sample was exchanged into NMR buffer ( $10 \mathrm{mM}$ sodium phosphate, $\mathrm{pH} 6.5,50$ $\mathrm{mM} \mathrm{NaCl}$ in $10 \%{ }^{2} \mathrm{H}_{2} \mathrm{O} / 90 \% \mathrm{H}_{2} \mathrm{O}$ or $100 \% 2 \mathrm{H}_{2} \mathrm{O}$ ) using a Centriprep (Amicon). NMR samples contain a 1:1 molar ratio of p53:NCBD, with the concentration of the proteins ranging from $\sim 0.5-1 \mathrm{mM}$.

\section{NMR Spectroscopy}

All NMR experiments were performed on Bruker 600, 750, 800, and $900 \mathrm{MHz}$ spectrometers at $298 \mathrm{~K}$. NMR spectra were referenced to external DSS. NMR data processing and analysis were performed using NMRPipe (45) and NMRView (46). Backbone resonance assignment was accomplished using standard HNCA (47), $\mathrm{HNCACB}(48)$, and $\mathrm{CBCA}(\mathrm{CO}) \mathrm{NH}$ (47) triple-resonance experiments. Assignments of side chain resonances were made using 3D HCCH-COSY and HCCH-TOCSY experiments (49). Torsion angle restraints were derived from 3D HACAHB-COSY (50), HNHB (51), and HNHA (52) datasets. Distance restraints were derived from 3D ${ }^{15} \mathrm{~N}$-edited NOESY-HSQC $\left(\tau_{\mathrm{m}}=100\right.$ and $\left.150 \mathrm{~ms}\right)$ and $13 \mathrm{C}$-edited NOESY-HSQC ( $\tau_{\mathrm{m}}=100$ and $\left.150 \mathrm{~ms}\right)$ spectra. Intermolecular NOEs used in structure calculations were obtained from ${ }^{13} \mathrm{C}$-filtered, ${ }^{12} \mathrm{C}$ edited NOESY-HSQC and ${ }^{13} \mathrm{C}$-edited, ${ }^{12} \mathrm{C}$-filtered NOESY-HSQC $\left(\tau_{\mathrm{m}}=200 \mathrm{~ms}\right)$ experiments.

\section{Structure Calculations and Analyses}

A total of 65 unambiguous intermolecular NOEs were manually assigned and used for the structure calculations. Initial structure calculations were carried out with the programs CYANA (53) with CANDID (54). Hydrogen bonds for the helical regions of the NCBD were added to facilitate the automated assignment of additional NOE restraints by CANDID, but were removed during the structure refinement. Chemical shift-based restraints from TALOS (55) were included only for helical regions. Based on the coupling constants and NOE patterns, 14 side chain $\chi_{1}$ angles were restrained to $\left(-60^{\circ}, 60^{\circ}, 180^{\circ}\right) \pm 30^{\circ}$.

The initial set of 100 structures generated in CYANA 2.1 with the redundant dihedral angle constraints (REDAC) (56) was further refined by molecular dynamics calculations with AMBER9 under in vacuo conditions with $20 \%$ reduced charges (57). Iterative refinement and editing of the distance restraints based on the NOESY spectra to remove incorrect and ambiguous assignments reduced the number of restraints. Final refinement in AMBER9 incorporated the generalized Born solvent model (58). Force constants were $20 \mathrm{kcal} \mathrm{mol}^{-1}$ $\AA^{-2}$ for NOE restraints and $200 \mathrm{kcal} \mathrm{mol}^{-1} \mathrm{rad}^{-2}$ for dihedral angle restraints. The 20 structures with the lowest generalized Born AMBER energy were chosen for analysis using 
the program PROCHECK-NMR (59). Figures were prepared using the program MOLMOL (60).

\section{Spin Labeling Experiment}

A spin-labeled NMR sample was prepared in NMR buffer at a molar ratio of 1:1 for Nterminal spin labeled $\left[{ }^{15} \mathrm{~N}\right] \mathrm{p} 53(13-61)$ and unlabeled NCBD. For measurements of paramagnetic relaxation enhancement, ${ }^{1} \mathrm{H}^{-15} \mathrm{~N}$ HSQC spectra were recorded in the presence and absence of ascorbic acid. Reduction of the spin label to its diamagnetic state was achieved by addition of a 5-fold molar excess of ascorbic acid and incubation overnight. The intensity ratios of ${ }^{1} \mathrm{H}_{-}{ }^{15} \mathrm{~N}$ HSQC peaks were calculated as $I_{\text {para }} / I_{\text {dia }}$, where $I_{\text {para }}$ is the resonance intensity of the spin-labeled sample, $I_{\mathrm{dia}}$ is the resonance intensity of the spinlabeled sample following reduction by ascorbic acid

\section{RESULTS}

In order to dissect the interactions between $\mathrm{p} 53 \mathrm{TAD}$ and $\mathrm{NCBD}$, we prepared $\mathrm{p} 53$ constructs encompassing the full-length TAD (residues 1-61) and various truncated peptides (residues 13-61, 25-61, 14-28, and 38-61; the latter two peptides encompass the isolated $\mathrm{AD} 1$ and $\mathrm{AD} 2$ subdomains, respectively). The interactions between the p53 TAD constructs and the NCBD were investigated both by ITC and NMR and the measured dissociation constants $\left(K_{\mathrm{d}}\right)$ are summarized in Fig. 1a. The longest peptide, p53(1-61), binds with highest affinity while the $\mathrm{N}$-terminal truncated peptides p53(13-61) and p53(25-61) bind about 2-3 fold more weakly. The peptides representing the isolated AD1 and AD2 subdomains bind approximately 170 - and 8-fold more weakly than p53(1-61), respectively. These results indicate that binding of the p53 TAD to the NCBD is dominated by the AD2 region, although the AD1 region does contribute somewhat to the overall binding affinity.

\section{Mutual Synergistic Folding between p53 TAD and CBP NCBD}

The p53 TAD in the unbound state is intrinsically disordered, as evidenced by the limited dispersion in the ${ }^{1} \mathrm{H}_{-}{ }^{15} \mathrm{~N}$ HSQC spectrum (Fig. 2) and by a circular dichroism spectrum that is characteristic of a random coil (Supplementary Fig. S2). The free NCBD domain is not entirely unstructured but forms a helical state with the characteristics of a molten globule (42). Formation of a complex is accompanied by a significant increase in dispersion in of the ${ }^{1} \mathrm{H}_{-}{ }^{15} \mathrm{~N}$ HSQC spectra (Fig. 2) and increased helicity in CD spectra of the bound state (Supplementary Fig. S2), showing that both proteins fold upon binding. Binding to the NCBD also results in shifts of the ${ }^{13} \mathrm{C} \alpha$ resonances in two regions of $\mathrm{p} 53$, confirming formation of helical structure between residues 19-24 and 47-53 in the complex (Supplementary Fig. S3a). Slight increases in helical structure are also observed for the NCBD in the bound state (Supplementary Fig. S3b).

\section{Structure Determination}

The structure of the complex formed between the NCBD and p53(13-61) was determined using constraints derived from heteronuclear multi-dimensional NMR experiments. We opted to use the N-terminal truncated p53 TAD (residues 13-61) rather than the full-length TAD for structure determination because the affinities of p53(1-61) and p53(13-61) for NCBD are similar (differing by less than 2-fold); residues N-terminal to Thr18 display anomalously small ${ }^{1} \mathrm{H}_{-}{ }^{15} \mathrm{~N}$ heteronuclear NOEs in the complex (Fig. 1b) and appear not to interact with the NCBD. In order to minimize spectral overlap and simplify the assignment process, complexes were formed with only one component ${ }^{15} \mathrm{~N}$ - or ${ }^{13} \mathrm{C} /{ }^{15} \mathrm{~N}$ - labeled and the other unlabeled. In contrast to the free proteins, the NMR spectra of the p53 TAD and NCBD in the complex are well dispersed and allow for nearly complete resonance assignment ( $95 \%$ of the ${ }^{1} \mathrm{H},{ }^{13} \mathrm{C}$, and ${ }^{15} \mathrm{~N}$ resonances). 
Initially, 24 spectroscopically unambiguous intermolecular NOEs between the p53 TAD and the NCBD were assigned manually and used as restraints in the first round of CYANA structure calculations. An additional 41 intermolecular NOE restraints were assigned using the program CANDID. These CANDID-assigned intermolecular NOEs were subsequently checked and verified against the filtered NOESY spectra $\left({ }^{13} \mathrm{C}\right.$-filtered, ${ }^{12} \mathrm{C}$-edited NOESYHSQC and ${ }^{13} \mathrm{C}$-edited, ${ }^{12} \mathrm{C}$-filtered NOESY-HSQC) of both ${ }^{13} \mathrm{C} /{ }^{15} \mathrm{~N}$ p53 TAD:unlabeled NCBD and ${ }^{13} \mathrm{C} /{ }^{15} \mathrm{~N}$ NCBD:unlabeled p53 TAD complexes. A total of 1503 distance restraints and 132 torsion angle restraints were used in the final rounds of structure calculation and refinement. The 20 structures with lowest AMBER energies are shown in Fig. $3 \mathrm{a}$ and the structural statistics are summarized in Table 1. All residues in structured regions have low RMSD values (backbone $=0.61 \AA$ and all heavy atoms $=0.93 \AA$ ). There are no dihedral angle violations in the final ensemble of 20 structures, the maximum NOE distance violation is 0.25 , and all residues are in allowed regions of the Ramachandran plot.

\section{Overall Structure of the p53 TAD:NCBD Complex}

In the bound state, $\mathrm{p} 53$ TAD comprises two helical regions (denoted $\mathrm{P} \alpha 1$ and $\mathrm{P} \alpha 2$ ) extend from Phe19 to Leu25 and Pro47 to Trp53, respectively (Fig. 3b). The N- and C-terminal residues and residues around 27-39, which form a long loop between the P $\alpha 1$ and $\mathrm{P} \alpha 2$ helices, are disordered in the structure ensemble (Fig. 3a) in accord with their small heteronuclear NOEs (Fig. 1b), sharp resonances, and the lack of intermolecular NOEs for these residues.

The NCBD is composed of three $\alpha$-helices (C $\alpha 1$ : residues 2066-2075, C $\alpha 2$ : residues 20812092, and Ca3: residues 2095-2105) that pack to expose a broad hydrophobic groove into which helices $P \alpha 1$ and $P \alpha 2$ of p53 dock (Figs. 3b, 3c). The helices of p53 are close to each other in the complex, as evidenced by long range intramolecular NOEs between these regions and spin labeling experiments; a paramagnetic spin label attached through a cysteine residue at the $\mathrm{N}$-terminus of p53(13-61) causes broadening of resonances from residues in both P $\alpha 1$ and P $\alpha 2$ (Supplementary Fig. S4). The residue 27-39 loop of p53 wraps around helix $\mathrm{C} \alpha 3$ of the NCBD but appears to form no persistent contacts since it gives rise to no detectable intermolecular NOEs.

\section{p53 TAD:NCBD Interface}

The interface between the NCBD and the P $\alpha 1$ and P $\alpha 2$ helices of the p53 TAD is predominantly hydrophobic (Fig. 3c). Several basic residues are located around this hydrophobic surface and form complementary electrostatic interactions with acidic side chains on the p53 TAD. Helix P $\alpha 1$ of p53 occupies a region of the hydrophobic groove formed by L2068, L2072, L2097, and F2101 in the C $\alpha 1$ and C $\alpha 3$ helices of the NCBD. In addition, L26 of p53 makes a hydrophobic contact with A2100 and F2101 of NCBD (Fig. 3d). Four hydrophobic residues (M40, L43, M44, and L45) of p53 bind to the top of the hydrophobic groove formed by $\mathrm{C} \alpha 2$ and $\mathrm{C} \alpha 3$, packing against L2088, M2098, F2101, and I2102 of the NCBD (Fig. 3e). The second helix of p53 (P $\alpha 2)$ interacts with the side chains of L2071, L2072, L2075, V2087, L2091, and F2101 of the NCBD. The two Trp and two Phe residues in $P \alpha 1$ and $P \alpha 2$ are deeply buried within the hydrophobic interface between $\mathrm{p} 53$ and the NCBD. Long-range NOEs between P $\alpha 1$ and P $\alpha 2$, including F19-W53, L22-Q52, and L22-W53, were detected in the NOESY-HSQC spectra, providing direct spectroscopic confirmation of the proximal location of these helices.

The total buried hydrophobic surface area is $880 \AA^{2}$, of which $580 \AA^{2}$ is contributed by helix $\mathrm{P} \alpha 2$ and the neighboring residues 40-45; the remainder of the hydrophobic surface is buried by helix P $\alpha 1$ of p53. There is a salt bridge between D49 of p53 TAD and R2105 of NCBD which may contribute to binding specificity (Fig. 3e). Substitution of D49 by Ala decreases 
the binding affinity only slightly but significantly changes the energetics of the interaction between p53 TAD and NCBD (Table 2). Other short truncated p53 TADs containing D49 showed similar favorable enthalpy changes $(\Delta \mathrm{H})$ to p53 (13-61) (Table 2), indicating that the salt bridge between D49 of the p53 TAD and R2106 of NCBD does play a role in complex formation.

\section{DISCUSSION}

\section{Structure of p53 TAD}

The N-terminal region of p53 consists of two domains, the transcriptional activation domain (TAD) and a proline-rich domain (PRD), which are directly involved in protein-protein interactions, post-translational modifications, and regulation of activity of p53. The PRD has a tendency to adopt polyproline II structure in the full-length p53, as shown by NMR and SAXS (61). The unbound TAD is intrinsically disordered, although transient local structures and long range interactions have been observed in NMR experiments $(34,62)$. The TAD contains two subdomains, $\mathrm{AD} 1$ and $\mathrm{AD} 2$, which mediate the interactions of $\mathrm{p} 53$ with MDM2 and CBP/p300 $(35,38,63)$. The AD1 region is the primary binding site for MDM2 and folds into an amphipathic $\alpha$-helix (residues 18-26) upon complex formation (Fig. 4a). The p53 TAD interacts with replication protein A (RPA) and the Tfb1/p62 subunit of TFIIH by way of the AD2 subdomain. Binding to RPA causes AD2 to fold to form two short helices, located between residues 41 and 44 and between residues 47 and 55 (Fig. 4a). Residues 47-55 also fold into a helical structure when AD2 binds to the Tfb1 subunit of TFIIH (Fig. 4a). Binding to CBP/p300 is also dominated by interactions with AD2; the p53 TAD can bind simultaneously to MDM2 and to the TAZ1, TAZ2, KIX, or NCBD domains of $\mathrm{CBP} / \mathrm{p} 300$ through the $\mathrm{AD} 1$ and $\mathrm{AD} 2$ motifs, respectively, to form a ternary complex (38).

To date, all structures reported for the p53 TAD have been for the isolated AD1 or AD2 subdomains only. In the present work, we determined structures of the full-length TAD, encompassing both the AD1 and AD2 regions, in complex with the NCBD domain of CBP. Both AD1 and AD2 interact directly with the NCBD, and each subdomain folds upon binding to form a helical motif ( $\mathrm{P} \alpha 1$, residues 19-25 and $\mathrm{P} \alpha 2$, residues 47-53). Residues 40-45 of p53, which adopt helical structure in the RPA complex, also contribute to the binding to NCBD, although apparently without formation of regular secondary structure. The helical regions of the p53 TAD are very similar in the complexes with the NCBD, MDM2, RPA, and Tfb1 (Fig. 4a), suggesting that formation of a conserved local structure is a feature of $\mathrm{p} 53$ recognition.

\section{Structure of NCBD}

The free CBP NCBD domain exhibits the spectroscopic and thermodynamic characteristics of a molten globule; although helices $\mathrm{C} \alpha 1$ and $\mathrm{C} \alpha 2$ are almost fully folded and helix $\mathrm{C} \alpha 3$ is partly formed in the free NCBD, the protein is not cooperatively folded and displays inherent flexibility $(15,42,64)$. Folding of the NCBD is induced upon interaction with its binding partners $(16,42)$. Although the NCBD is stabilized as a three-helix bundle in all of its complexes, the length of the helices and the packing topology differs significantly in complexes with different binding partners. In particular, the NCBD adopts a very different conformation in its complex with the globular protein IRF-3 (65) than it does when bound to the disordered interaction domains of the nuclear receptor coactivators ACTR and SRC1 $(42,65,66)$. Fig. $4 \mathrm{~b}$ shows a superposition of the NCBD structure in its complexes with the p53 TAD, ACTR, and SRC1. While the overall fold of the NCBD is similar in these three complexes (the backbone RMSD in the structured regions is $1.4 \AA$ ), some differences are observed in the length and orientation of helix $\mathrm{C} \alpha 3$. This helix is extended by an extra turn 
at the C-terminus in the ACTR complex, presumably as a consequence of extensive hydrophobic contacts with the third helix of ACTR (42). The angle between helices C $\alpha 2$ and $\mathrm{C} \alpha 3$ of the NCBD is larger $\left(67^{\circ}\right)$ in the p53 TAD complex than in the complex with ACTR $\left(58^{\circ}\right)$. This may reflect the fact that the two helices $(\mathrm{P} \alpha 1$ and $\mathrm{P} \alpha 2)$ of the p53 TAD are packed across the hydrophobic groove of NCBD, compared to a single helix lying along the groove in the ACTR and SRC1 complexes. In addition, the binding affinities are very different for the NCBD complexes with ACTR and the p53 TAD $\left(K_{\mathrm{d}}=0.034\right.$ vs. $\left.3.1 \mu \mathrm{M}\right)$. The C $\alpha 2$ helix is observed to be slightly shorter in the ACTR complex than in the p53 or SRC1 structures; however, this difference probably reflects lack of resonance assignments and a corresponding lack of restraints for the poly-Q segment (residues 2082-2086) of the ACTR complex, rather than real structural differences.

\section{Recognition of the NCBD by the p53 TAD}

A helical region containing the LXXLL motif mediates the interactions among CBP/p300, p160 coactivators, and the ligand-binding domains of nuclear receptors (67-70). Similar motifs, with $\varphi \varphi \mathrm{XX} \varphi$ or $\varphi \mathrm{XX} \varphi \varphi$ sequence patterns, where $\varphi$ is a bulky hydrophobic residues (commonly leucine) and $\mathrm{X}$ is any other residue, directly mediate the interaction between the NCBD and ACTR (Fig. 5) (42). These motifs are also found in the AD1 and AD2 regions of the p53 TAD (Fig. 5), although the mode of interaction with the NCBD is different from that of ACTR. The hydrophobic residues in AD2 (M40, L43, M44, L45, I50, W53, and F54) are directly involved in interactions with the NCBD, in the deep groove formed between helices $\mathrm{C} \alpha 1-\mathrm{C} \alpha 2$ and helix $\mathrm{C} \alpha 3$. Residues $40-45$ appear to be less well ordered and have a smaller heteronuclear NOE than residues in helices $\mathrm{P} \alpha 1$ and $\mathrm{P} \alpha 2$, but this region of the $\mathrm{p} 53$ backbone exhibits a tendency towards a distorted helical conformation in the ensemble of p53:NCBD structures. In addition, F19, W23, and L26 in AD1 region also interact with the hydrophobic surface formed between helices $\mathrm{C} \alpha 1$ and $\mathrm{C} \alpha 3$. The point mutations L22Q or W23S do not significantly impair p53 binding to the NCBD domain (26), indicating that the hydrophobic residues in AD2 are essential to stabilize the hydrophobic core of the complex. L2068 and L2071 of the NCBD make a hydrophobic contact with F19 and F54, respectively, of the $\mathrm{p} 53$ TAD. The double mutation in which both NCBD residues are replaced by alanine (L2068A, L2071A) has a significant effect on p53 binding (26), indicating that F19 and F54 are also critical for complex formation. Mutation of hydrophobic residues in AD2 impairs binding of p53 to the NCBD; the double mutant W53Q/F54S binds with about threefold lower affinity than the wild type TAD (63). Simultaneous mutation of both AD1 and AD2 (L22Q/W23S and W53Q/F54S) has much more deleterious effects on binding (63), indicating that the hydrophobic residues in AD1 and AD2 are synergistically involved in the binding to the NCBD. These data are fully consistent with our structure of the p53 TAD:NCBD complex, where both AD1 and AD2 simultaneously interact with the NCBD domain.

Although the interface between the p53 TAD and the NCBD is predominantly hydrophobic, a salt bridge (between D49 of p53 and R2105 of the NCBD) also contributes to the interaction. In the ACTR:NCBD complex, R2105 of NCBD makes a salt bridge with D1068 of ACTR to provide binding specificity but does not contribute significantly to binding affinity or stability $(15,42)$. The single substitution of the salt bridge (NCBD R2105L) has a relatively minor effect on the $K_{\mathrm{d}}$ of the complex, but causes a reduction in the favorable enthalpy change upon binding. In the complex between the p53 TAD and NCBD, we observe similar energetic effects from disruption of the salt bridge by substitution of D49 of p53 by alanine; the mutation has only a minor effect on the $K_{\mathrm{d}}$ but causes 2 -fold reduction in the favorable enthalpy change that accompanies binding $(\Delta \mathrm{H}=-2.1 \mathrm{kcal} / \mathrm{mol}$ for D49A versus $\Delta \mathrm{H}=-4.4 \mathrm{kcal} / \mathrm{mol}$ for wild-type $\mathrm{p} 53$ ). Therefore, as with ACTR, the salt bridge in the p53 TAD:NCBD complex may contribute to specificity rather than binding affinity. 
Thus, it appears that R2105 of the NCBD may play a general role in target selection among the many nuclear proteins that use amphipathic helical motifs for recognition.

\section{Structural and Functional Malleability of p53}

The human p53 contains large disordered regions including N-terminal and C-terminal domains which interact with more than one protein. Short recognition domains embedded in disordered regions in eukaryotic transcriptional complexes can lead to structural and functional malleability that provides adaptability to recognize multiple targets having different structures (71). The $\mathrm{N}$-terminal transactivation domain of p53 interacts with several partners including CBP/p300, RPA, Tfb1 and MDM2. Although the two short binding domains (AD1 and AD2) in the p53 TAD are structurally homologous motifs that form an amphipathic helix upon binding to their target proteins, their functions are distinct. Binding of the p53 TAD to CBP/p300, RPA and Tfb1 is dominated by interaction with AD2. In contrast, binding of p53 TAD to MDM2 is mediated primarily by AD1; however, upon phosphorylation of $\mathrm{p} 53$ at Thr18 in response to DNA damage, MDM2 is released and the p53 TAD binds preferentially to the NCBD and other domains of CBP/p300 (38). The present structure shows that, in the absence of MDM2, both the AD1 and AD2 motifs of the p53 TAD interact with the NCBD domain, and that the 13 residues between them remain disordered in the bound form. While binding is dominated by the AD2 motif $\left(K_{\mathrm{d}}=5-14 \mu \mathrm{M}\right.$ for peptides containing only $\mathrm{AD} 2$, compared to $K_{\mathrm{d}}>300 \mu \mathrm{M}$ for the isolated AD1 peptide, Fig. 1), both AD1 and AD2 contribute to the overall affinity for binding to the NCBD. AD1 and AD2 essentially function as clamps, connected by a disordered linker that does not make direct contacts with the NCBD. A clamp model of binding has been observed for other intrinsically disordered proteins, leading to an enhancement of binding affinity compared to the isolated motifs and providing flexibility and adaptability in molecular interactions (72). In the case of $\mathrm{p} 53$, the presence of two interaction motifs in the intrinsically disordered TAD imparts the flexibility to form ternary complexes with MDM2 and CBP/p300 domains, or to bind with enhanced affinity through clamp-like interactions with the NCBD or other CBP/ p300 domains (38).

\section{Supplementary Material}

Refer to Web version on PubMed Central for supplementary material.

\section{Acknowledgments}

We thank Josephine Ferreon for providing ITC data on the interaction of NCBD with p53(1-61). Mindy Landes and Euvel Manlapaz for help with sample preparation and John Chung and Gerard Kroon for expert assistance with NMR data collection.

\section{Abbreviations}

$\begin{array}{ll}\text { CD } & \text { circular dichroism } \\ \text { CBP } & \text { CREB binding protein } \\ \text { CREB } & \text { cyclic-AMP response element binding protein } \\ \text { DSS } & \text { sodium 2,2-dimethyl-2,-silapentane-5-sulfonate } \\ \text { HDM2 } & \text { human homolog of MDM2 } \\ \text { MDM2 } & \text { mouse double minute protein } 2 \\ \text { HSQC } & \text { heteronuclear single quantum coherence }\end{array}$




$\begin{array}{ll}\text { IBiD } & \text { IRF-3 binding domain } \\ \text { IRF } & \text { interferon regulatory factor } \\ \text { ITC } & \text { isothermal titration calorimetry } \\ \text { MTSL } & \text { (1-oxyl-2,2,5,5-tetramethyl- } \Delta^{3} \text {-pyrrolin-3-yl)methyl methanethiosulfonate } \\ \text { NCBD } & \text { nuclear receptor coactivator binding domain } \\ \text { NMR } & \text { nuclear magnetic resonance } \\ \text { SID } & \text { SRC1 interaction domain } \\ \text { SRC1 } & \text { steroid receptor coactivator 1 } \\ \text { TAD } & \text { transactivation domain } \\ \text { TAZ } & \text { transcriptional adapter zinc finger }\end{array}$

\section{References}

1. Greenblatt MS, Bennett WP, Hollstein M, Harris CC. Mutations in the p53 tumor suppressor gene: clues to cancer etiology and molecular pathogenesis. Cancer Res. 1994; 54:4855-4878. [PubMed: 8069852]

2. Soussi T, Beroud C. Assessing TP53 status in human tumours to evaluate clinical outcome. Nat Rev Cancer. 2001; 1:233-240. [PubMed: 11902578]

3. Moll UM, Petrenko O. The MDM2-p53 interaction. Mol Cancer Res. 2003; 1:1001-1008. [PubMed: 14707283]

4. Brooks CL, Gu W. p53 ubiquitination: Mdm2 and beyond. Mol Cell. 2006; 21:307-315. [PubMed: 16455486]

5. Momand J, Zambetti GP, Olson DC, George D, Levine AJ. The mdm-2 oncogene product forms a complex with the p53 protein and inhibits p53-mediated transactivation. Cell. 1992; 69:1237-1245. [PubMed: 1535557]

6. Thut CJ, Goodrich JA, Tjian R. Repression of p53-mediated transcription by MDM2: a dual mechanism. Genes Dev. 1997; 11:1974-1986. [PubMed: 9271120]

7. Toledo F, Wahl GM. Regulating the p53 pathway: in vitro hypotheses, in vivo veritas. Nat Rev Cancer. 2006; 6:909-923. [PubMed: 17128209]

8. Toledo F, Krummel KA, Lee CJ, Liu CW, Rodewald LW, Tang M, Wahl GM. A mouse p53 mutant lacking the proline-rich domain rescues Mdm4 deficiency and provides insight into the Mdm2Mdm4-p53 regulatory network. Cancer Cell. 2006; 9:273-285. [PubMed: 16616333]

9. Lambert PF, Kashanchi F, Radonovich MF, Shiekhattar R, Brady JN. Phosphorylation of p53 serine 15 increases interaction with CBP. J Biol Chem. 1998; 273:33048-33053. [PubMed: 9830059]

10. Dumaz N, Meek DW. Serine15 phosphorylation stimulates p53 transactivation but does not directly influence interaction with HDM2. EMBO J. 1999; 18:7002-7010. [PubMed: 10601022]

11. Sakaguchi K, Saito S, Higashimoto Y, Roy S, Anderson CW, Appella E. Damage-mediated phosphorylation of human p53 threonine 18 through a cascade mediated by a casein 1-like kinase. Effect on Mdm2 binding. J Biol Chem. 2000; 275:9278-9283. [PubMed: 10734067]

12. Dornan D, Hupp TR. Inhibition of p53-dependent transcription by BOX-I phospho-peptide mimetics that bind to p300. EMBO Rep. 2001; 2:139-144. [PubMed: 11258706]

13. Schon O, Friedler A, Bycroft M, Freund SM, Fersht AR. Molecular mechanism of the interaction between MDM2 and p53. J Mol Biol. 2002; 323:491-501. [PubMed: 12381304]

14. Goodman RH, Smolik S. CBP/p300 in cell growth, transformation, and development. Genes Devel. 2000; 14:1553-1577. [PubMed: 10887150]

15. Demarest SJ, Deechongkit S, Dyson HJ, Evans RM, Wright PE. Packing, specificity, and mutability at the binding interface between the p160 coactivator and CREB-binding protein. Protein Sci. 2004; 13:203-210. [PubMed: 14691235] 
16. Lin CH, Hare BJ, Wagner G, Harrison SC, Maniatis T, Fraenkel E. A small domain of cbp/p300 binds diverse proteins. solution structure and functional studies. Mol Cell. 2001; 8:581-590. [PubMed: 11583620]

17. Sheppard HM, Harries JC, Hussain S, Bevan C, Heery DM. Analysis of the steroid receptor coactivator 1 (SRC1)-CREB binding protein interaction interface and its importance for the function of SRC1. Mol Cell Biol. 2001; 21:39-50. [PubMed: 11113179]

18. Tang Y, Zhao W, Chen Y, Zhao Y, Gu W. Acetylation is indispensable for p53 activation. Cell. 2008; 133:612-626. [PubMed: 18485870]

19. Avantaggiati ML, Ogryzko V, Gardner K, Giordano A, Levine A, Kelly K. Recruitment of p300/ CBP in p53-dependent signal pathways. Cell. 1997; 89:1175-1184. [PubMed: 9215639]

20. Gu W, Shi XL, Roeder RG. Synergistic activation of transcription by CBP and p53. Nature. 1997; 387:819-823. [PubMed: 9194564]

21. Lill NL, Grossman SR, Ginsberg D, DeCaprio J, Livingston DM. Binding and modulation of p53 by p300/CBP coactivators. Nature. 1997; 387:823-827. [PubMed: 9194565]

22. Scolnick DM, Chehab NH, Stavridi ES, Lien MC, Caruso L, Moran E, Berger SL, Halazonetis TD CREB-binding protein and p300/CBP-associated factor are transcriptional coactivators of the p53 tumor suppressor protein. Cancer Res. 1997; 57:3693-3696. [PubMed: 9288775]

23. Grossman SR, Perez M, Kung AL, Joseph M, Mansur C, Xiao ZX, Kumar S, Howley PM, Livingston DM. p300/MDM2 complexes participate in MDM2-mediated p53 degradation. Mol Cell. 1998; 2:405-415. [PubMed: 9809062]

24. van Orden K, Giebler HA, Lemasson I, Gonzales M, Nyborg JK. Binding of p53 to the KIX domain of CREB binding protein - A potential link to human T-cell leukemia virus, type Iassociated leukemogenesis. J Biol Chem. 1999; 274:26321-26328. [PubMed: 10473588]

25. Wadgaonkar R, Collins T. Murine double minute (MDM2) blocks p53-coactivator interaction, a new mechanism for inhibition of p53-dependent gene expression. J Biol Chem. 1999; 274:13760 13767. [PubMed: 10318779]

26. Livengood JA, Scoggin KES, van Orden K, McBryant SJ, Edayathumangalam RS, Laybourn PJ, Nyborg JK. p53 transcriptional activity Is mediated through the SRC1-interacting domain of CBP/ p300. J Biol Chem. 2002; 277:9054-9061. [PubMed: 11782467]

27. Unger T, Mietz JA, Scheffner M, Yee CL, Howley PM. Functional domains of wild-type and mutant p53 proteins involved in transcriptional regulation, transdominant inhibition, and transformation suppression. Mol Cell Biol. 1993; 13:5186-5194. [PubMed: 8355677]

28. Chang J, Kim DH, Lee SW, Choi KY, Sung YC. Transactivation ability of p53 transcriptional activation domain is directly related to the binding affinity to TATA-binding protein. J Biol Chem. 1995; 270:25014-25019. [PubMed: 7559631]

29. Candau R, Scolnick DM, Darpino P, Ying CY, Halazonetis TD, Berger SL. Two tandem and independent sub-activation domains in the amino terminus of p53 require the adaptor complex for activity. Oncogene. 1997; 15:807-816. [PubMed: 9266967]

30. Zhu J, Zhou W, Jiang J, Chen X. Identification of a novel p53 functional domain that is necessary for mediating apoptosis. J Biol Chem. 1998; 273:13030-13036. [PubMed: 9582339]

31. Ayed A, Mulder FA, Yi GS, Lu Y, Kay LE, Arrowsmith CH. Latent and active p53 are identical in conformation. Nat Struct Biol. 2001; 8:756-760. [PubMed: 11524676]

32. Dawson R, Muller L, Dehner A, Klein C, Kessler H, Buchner J. The N-terminal domain of p53 is natively unfolded. J Mol Biol. 2003; 332:1131-1141. [PubMed: 14499615]

33. Botuyan MVE, Momand J, Chen Y. Solution conformation of an essential region of the p53 transactivation domain. Fold Design. 1997; 2:331-342.

34. Lee H, Mok KH, Muhandiram R, Park KH, Suk JE, Kim DH, Chang J, Sung YC, Choi KY, Han $\mathrm{KH}$. Local structural elements in the mostly unstructured transcriptional activation domain of human p53. J Biol Chem. 2000; 275:29426-29432. [PubMed: 10884388]

35. Kussie PH, Gorina S, Marechal V, Elenbaas B, Moreau J, Levine AJ, Pavletich NP. Structure of the MDM2 oncoprotein bound to the p53 tumor suppressor transactivation domain. Science. 1996; 274:948-953. [PubMed: 8875929]

36. Bochkareva E, Kaustov L, Ayed A, Yi GS, Lu Y, Pineda-Lucena A, Liao JCC, Okorokov AL, Milner J, Arrowsmith CH, Bochkarev A. Single-stranded DNA mimicry in the p53 transactivation 
domain interaction with replication protein A. Proc Natl Acad Sci USA. 2005; 102:15412-15417. [PubMed: 16234232]

37. Di Lello P, Jenkins LMM, Jones TN, Nguyen BD, Hara T, Yamaguchi H, Dikeakos JD, Appella E, Legault $\mathrm{P}$, Omichinski JG. Structure of the Tfb1/p53 complex: insights into the interaction between the p62/Tfb1 subunit of TFIIH and the activation domain of p53. Mol Cell. 2006; 22:731-740. [PubMed: 16793543]

38. Ferreon JC, Lee CW, Arai M, Martinez-Yamout MA, Dyson HJ, Wright PE. Cooperative regulation of $\mathrm{p} 53$ by modulation of ternary complex formation with $\mathrm{CBP} / \mathrm{p} 300$ and HDM2. Proc Natl Acad Sci USA. 2009; 106:6591-6596. [PubMed: 19357310]

39. Ferreon JC, Martinez-Yamout MA, Dyson HJ, Wright PE. Structural basis for subversion of cellular control mechanisms by the adenoviral E1A oncoprotein. Proc Natl Acad Sci USA. 2009; 106:13260-13265. [PubMed: 19651603]

40. Feng H, Jenkins LMM, Durell SR, Hayashi R, Mazur SJ, Cherry S, Tropea JE, Miller M, Wlodawer A, Appella E, Bai Y. Structural basis for p300 Taz2-p53 TAD1 binding and modulation by phosphorylation. Structure. 2009; 17:202-210. [PubMed: 19217391]

41. Dornan D, Shimizu H, Burch L, Smith AJ, Hupp TR. The proline repeat domain of p53 binds directly to the transcriptional coactivator p300 and allosterically controls DNA-dependent acetylation of p53. Mol Cell Biol. 2003; 23:8846-8861. [PubMed: 14612423]

42. Demarest SJ, Martinez-Yamout M, Chung J, Chen H, Xu W, Dyson HJ, Evans RM, Wright PE. Mutual synergistic folding in recruitment of $\mathrm{CBP} / \mathrm{p} 300$ by $\mathrm{p} 160$ nuclear receptor coactivators. Nature. 2002; 415:549-553. [PubMed: 11823864]

43. Qin BY, Liu C, Srinath H, Lam SS, Correia JJ, Derynck R, Lin K. Crystal Structure of IRF-3 in complex with CBP. Structure. 2005; 13:1269-1277. [PubMed: 16154084]

44. Lee CW, Arai M, Martinez-Yamout MA, Dyson HJ, Wright PE. Mapping the ilnteractions of the p53 transactivation domain with the KIX domain of CBP. Biochemistry. 2009; 48:2115-2124. [PubMed: 19220000]

45. Delaglio F, Grzesiek S, Vuister GW, Guang Z, Pfeifer J, Bax A. NMRPipe: a multidimensional spectral processing system based on UNIX pipes. J Biomol NMR. 1995; 6:277-293. [PubMed: 8520220]

46. Johnson BA, Blevins RA. NMRView: A computer program for the visualization and analysis of NMR data. J Biomol NMR. 1994; 4:604-613.

47. Grzesiek S, Bax A. Improved 3D triple-resonance NMR techniques applied to a $31 \mathrm{kDa}$ protein. J Magn Reson. 1992; 96:432-440.

48. Wittekind M, Mueller L. HNCACB, a high-sensitivity 3D NMR experiment to correlate amideproton and nitrogen resonances with the alpha- and beta-carbon resonances in proteins. J Magn Reson. 1993; 101:201-205.

49. Bax A, Clore GM, Gronenborn AM. ${ }^{1} \mathrm{H}-{ }^{1} \mathrm{H}$ correlation via isotropic mixing of ${ }^{13} \mathrm{C}$ magnetization, a new three-dimensional approach for assigning ${ }^{1} \mathrm{H}$ and ${ }^{13} \mathrm{C}$ spectra of ${ }^{13} \mathrm{C}$-enriched proteins. $\mathrm{J}$ Magn Reson. 1990; 88:425-431.

50. Grzesiek S, Kuboniwa H, Hinck AP, Bax A. Multiple-quantum line narrowing for measurement of $\mathrm{H} \alpha-\mathrm{H} \beta \mathrm{J}$ coupling in isotopically enriched proteins. J Am Chem Soc. 1995; 117:5312-5315.

51. Archer SJ, Ikura M, Torchia DA, Bax A. An alternative 3D NMR technique for correlating backbone ${ }^{15} \mathrm{~N}$ with side chain $\mathrm{H} \beta$ resonances in larger proteins. J Magn Reson. 1991; 95:636-641.

52. Vuister GW, Bax A. Measurement of two- and three-bond proton to methyl-carbon J couplings in proteins uniformly enriched with ${ }^{13}$ C. J Magn Reson Series B. 1993; 102:228-231.

53. Güntert P. Automated protein structure calculation with CYANA. Meth Mol Biol. 2004; 278:353378.

54. Herrmann T, Güntert P, Wüthrich K. Protein NMR structure determination with automated NOE assignment using the new software CANDID and the torsion angle dynamics algorithm DYANA. J Mol Biol. 2002; 319:209-227. [PubMed: 12051947]

55. Cornilescu G, Delaglio F, Bax A. Protein backbone angle restraints from searching a database for chemical shift and sequence homology. J Biomol NMR. 1999; 13:289-302. [PubMed: 10212987] 
56. Güntert P, Wüthrich K. Improved efficiency of protein structure calculations from NMR data using the program DIANA with redundant dihedral angle constraints. J Biomol NMR. 1991; 1:447-456. [PubMed: 1841711]

57. Case DA, Cheatham TE III, Darden T, Gohlke H, Luo R, Merz KMJ, Onufriev A, Simmerling C, Wang B, Woods R. The Amber biomolecular simulation programs. J Comput Chem. 2005; 26:1668-1688. [PubMed: 16200636]

58. Tsui V, Case DA. Molecular simulations of nucleic acids using a generalized Born solvation model. J Am Chem Soc. 2000; 122:2489-2498.

59. Laskowski RA, Rullmann JAC, MacArthur MW, Kaptein R, Thornton JM. AQUA and PROCHECK-NMR: Programs for checking the quality of protein structures solved by NMR. J Biomol NMR. 1996; 8:477-486. [PubMed: 9008363]

60. Koradi R, Billeter M, Wüthrich K. MOLMOL: A program for display and analysis of macromolecular structures. J Mol Graphics. 1996; 14:51-55.

61. Wells M, Tidow H, Rutherford TJ, Markwick P, Jensen MR, Mylonas E, Svergun DI, Blackledge M, Fersht AR. Structure of tumor suppressor p53 and its intrinsically disordered N-terminal transactivation domain. Proc Natl Acad Sci USA. 2008; 105:5762-5767. [PubMed: 18391200]

62. Vise P, Baral B, Stancik A, Lowry DF, Daughdrill GW. Identifying long-range structure in the intrinsically unstructured transactivation domain of p53. Proteins: Structure, Function and Genetics. 2007; 67:526-530.

63. Teufel DP, Freund SM, Bycroft M, Fersht AR. Four domains of p300 each bind tightly to a sequence spanning both transactivation subdomains of p53. Proc Natl Acad Sci USA. 2007; 104:7009-7014. [PubMed: 17438265]

64. Ebert MO, Bae SH, Dyson HJ, Wright PE. NMR relaxation study of the complex formed between $\mathrm{CBP}$ and the activation domain of the nuclear hormone receptor coactivator ACTR. Biochemistry. 2008; 47:1299-1308. [PubMed: 18177052]

65. Lees MJ, Peet DJ, Whitelaw ML. Defining the role for XAP2 in stabilization of the dioxin receptor. J Biol Chem. 2003; 278:35878-35888. [PubMed: 12837759]

66. Waters L, Yue B, Veverka V, Renshaw P, Bramham J, Matsuda S, Frenkiel T, Kelly G, Muskett F, Carr M, Heery DM. Structural diversity in p160/CREB-binding protein coactivator complexes. J Biol Chem. 2006; 281:14787-14795. [PubMed: 16540468]

67. Heery DM, Kalkhoven E, Hoare S, Parker MG. A signature motif in transcriptional co-activators mediates binding to nuclear receptors. Nature. 1997; 387:733-736. [PubMed: 9192902]

68. McInerney EM, Rose DW, Flynn SE, Westin S, Mullen TM, Krones A, Inostroza J, Torchia J, Nolte RT, Assa-Munt N, Milburn MV, Glass CK, Rosenfeld MG. Determinants of coactivator LXXLL motif specificity in nuclear receptor transcriptional activation. Genes Devel. 1998; 12:3357-3368. [PubMed: 9808623]

69. Voegel JJ, Heine MJ, Tini M, Vivat V, Chambon P, Gronemeyer H. The coactivator TIF2 contains three nuclear receptor-binding motifs and mediates transactivation through CBP bindingdependent and -independent pathways. EMBO J. 1998; 17:507-519. [PubMed: 9430642]

70. Perissi V, Staszewski LM, McInerney EM, Kurokawa R, Krones A, Rose DW, Lambert MH, Milburn MV, Glass CK, Rosenfeld MG. Molecular determinants of nuclear receptor-corepressor interaction. Genes Dev. 1999; 13:3198-3208. [PubMed: 10617569]

71. Fuxreiter M, Tompa P, Simon I, Uversky VN, Hansen JC, Asturias FJ. Malleable machines take shape in eukaryotic transcriptional regulation. Nat Chem Biol. 2008; 4:728-737. [PubMed: 19008886]

72. Tompa P, Fuxreiter M. Fuzzy complexes: polymorphism and structural disorder in protein-protein interactions. Trends Biochem Sci. 2008; 33:2-8. [PubMed: 18054235] 
a

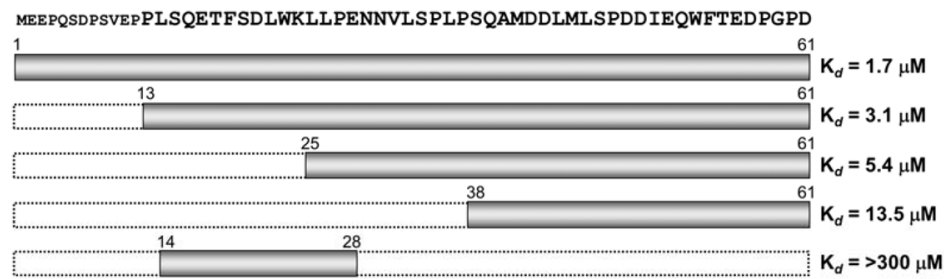

b

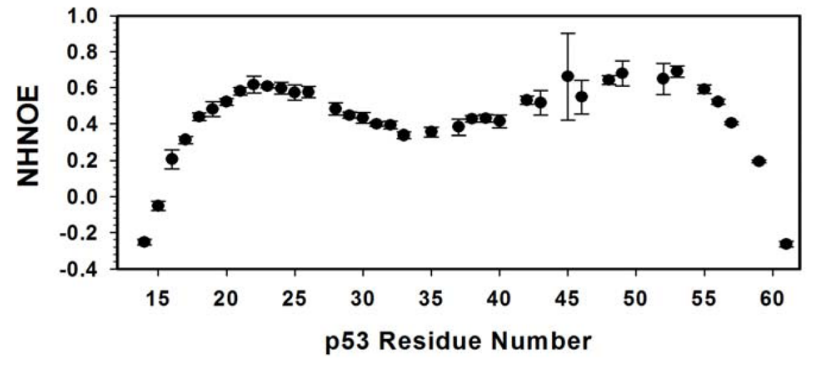

Figure 1.

Binding affinity and binding region of p53 TAD domains to NCBD. (a) Schematic drawing of various p53 TAD domains. The dissociation constants $\left(\mathrm{K}_{d}\right)$ of each domain are indicated. (b) Heteronuclear $\left[{ }^{1} \mathrm{H}\right]-{ }^{15} \mathrm{~N}$ NOE for $\left[{ }^{15} \mathrm{~N}\right] \mathrm{p} 53(13-61)$ bound to NCBD. 

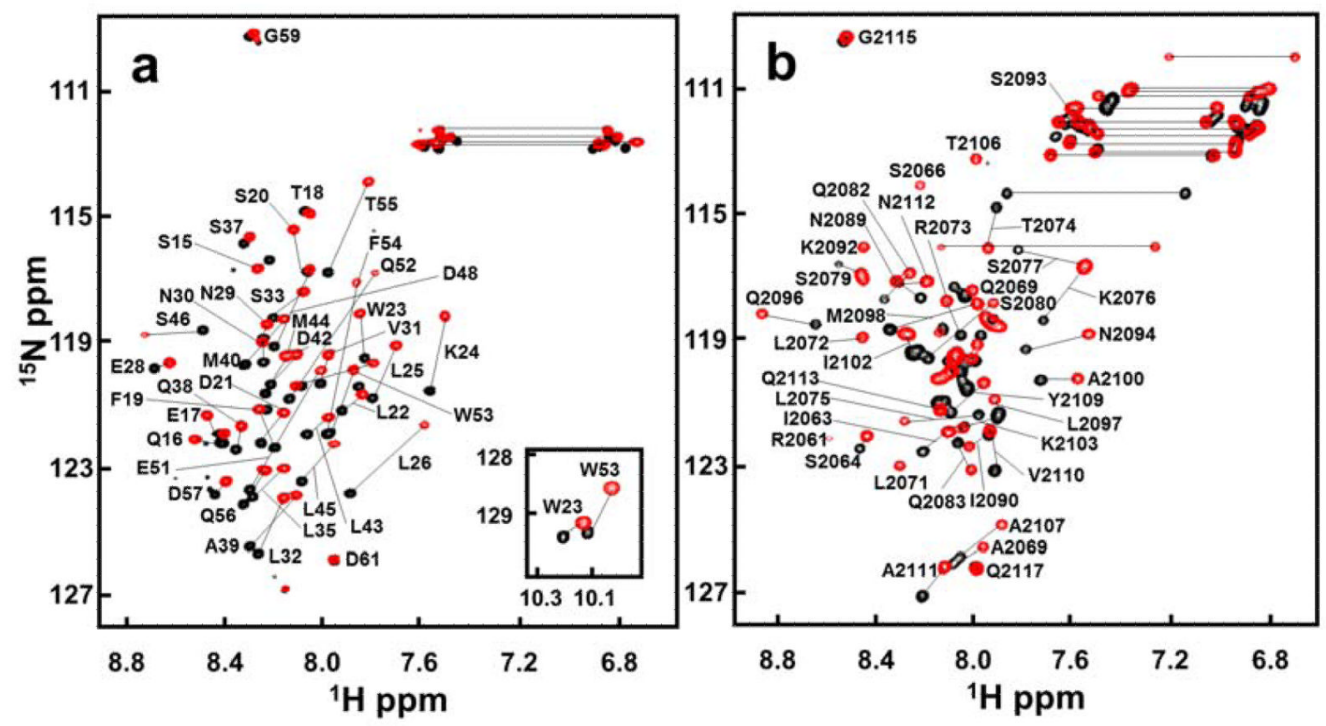

Figure 2.

${ }^{1} \mathrm{H}_{-}{ }^{15} \mathrm{~N}$ HSQC spectra of p53 TAD and NCBD. (a) $\left[{ }^{15} \mathrm{~N}\right] \mathrm{p} 53(13-61)$ free (black) and in the presence (1:1) of unlabeled NCBD (red). Peaks from two tryptophan side-chains are shown in the inset spectrum. (b) $\left[{ }^{15} \mathrm{~N}\right] \mathrm{NCBD}$ free (black) and in the presence $(1: 1)$ of unlabeled p53(13-61) (red). 

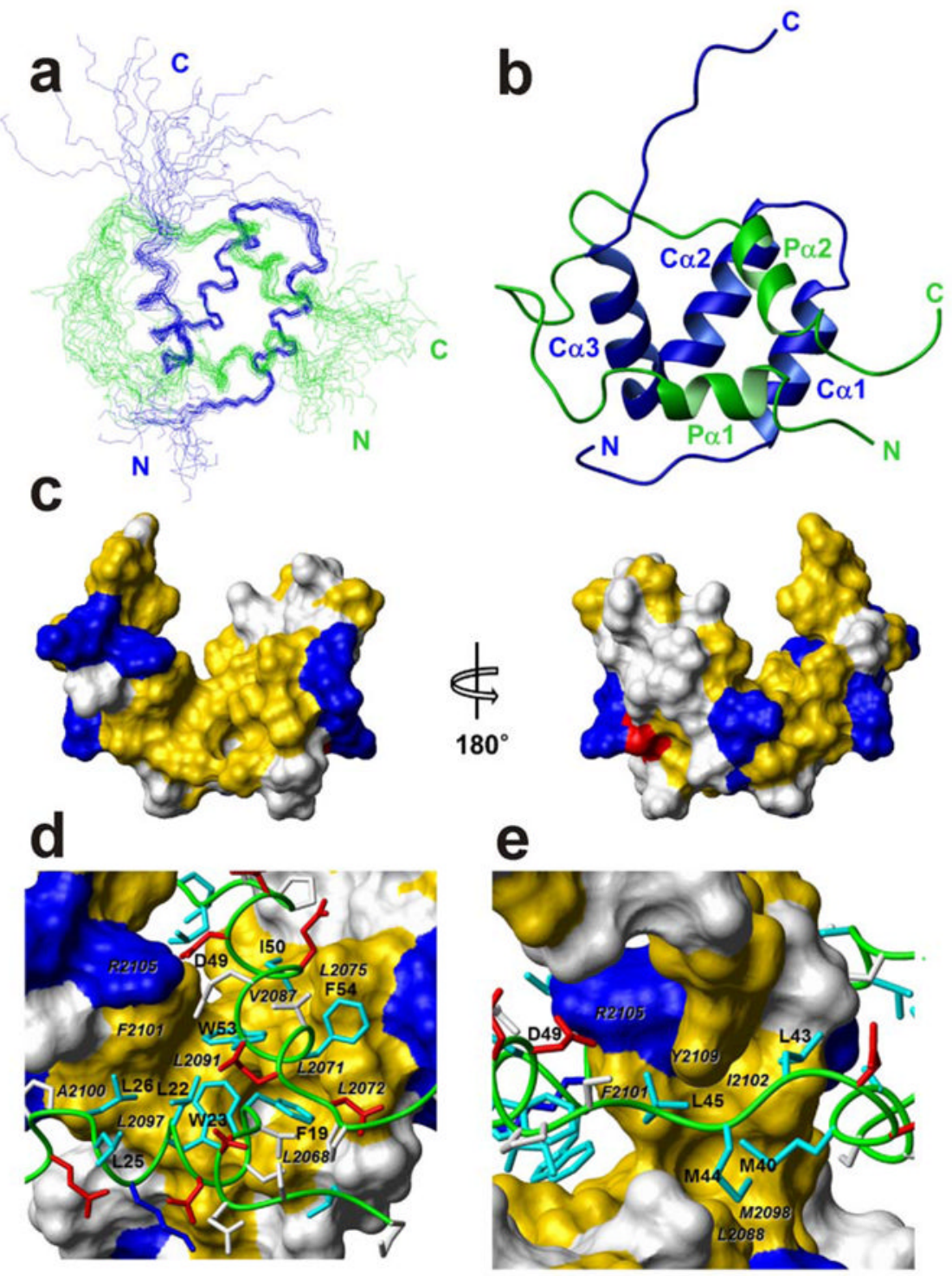

Figure 3.

Solution structure of the p53 TAD:NCBD complex. (a) The best 20 structures superposed on backbone heavy atoms in ordered regions. The p53 TAD backbone is shown in green, $\mathrm{NCBD}$ in blue, and the $\mathrm{N}$ and $\mathrm{C}$ termini of each chain are labeled in the corresponding colors. (b) Ribbon representation, in the same orientation and color as (a) Helices Pa1-2 and $\mathrm{C} \alpha 1-3$, and N and C termini are labeled. (c) Surface representation of NCBD domain in complex with p53 TAD. The left and right figures are rotated $180^{\circ}$ relative to one another about a vertical axis. Hydrophobic residues (Ala, Met, Leu, Ile, Val, Phe, and Tyr) are colored yellow; positively charged (Arg and Lys) and negatively charged (Asp) residues are colored blue and red, respectively. The other residues are colored white. (d) and (e) Binding site for $\mathrm{P} \alpha 1$ and $\mathrm{P} \alpha 2$ and extended region of 53 TAD (green tube) in the hydrophobic grooves on the surface of NCBD. The surfaces of interacting hydrophobic side chains from NCBD are labeled in black italic characters. The side chains of basic and acidic residues of p53 TAD are shown in blue and red, respectively. The side chains of hydrophobic residues of p53 TAD are shown in cyan and labeled. The side chains of other residues are colored white. 

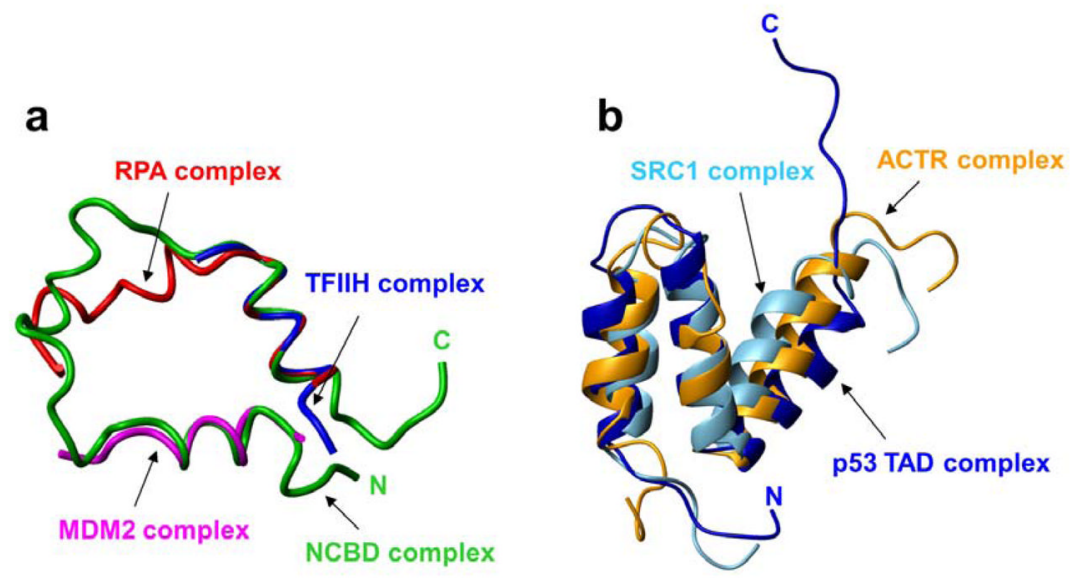

Figure 4.

Superposition of p53 TAD and NCBD structures. (a) The backbone of the p53 TAD in the complex with NCBD is shown as a green tube. The p53 TAD structures in the complex with other proteins are aligned on the backbone heavy atoms of $\mathrm{P} \alpha 1$ and $\mathrm{P} \alpha 2$ of $\mathrm{p} 53$ TAD in the complex with NCBD. The AD1 region of the p53 TAD in the complex with MDM2 is shown in magenta. The AD2 region of the p53 TAD in the complexes with RPA and TFIIH is shown in red and blue, respectively. (b) Superposition of the structures adopted by the NCBD in complexes with the p53 TAD (blue), ACTR (orange), and SRC1 (cyan). The NCBD backbone is shown as a ribbon, and the structures are superimposed on the backbone heavy atoms of residues in the $\mathrm{C} \alpha 1$ and $\mathrm{C} \alpha 2$ helices of the NCBD. 


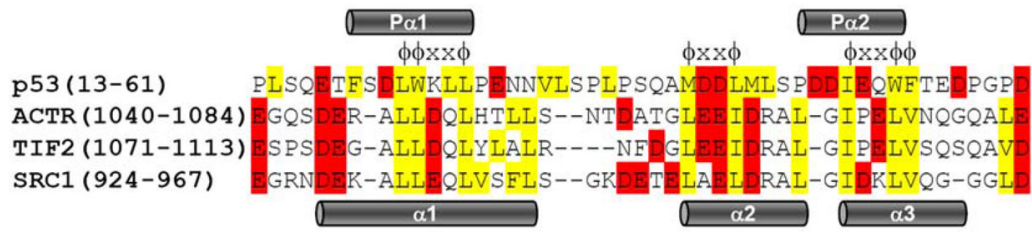

Figure 5.

Alignment of the amino acid sequences of NCBD binding proteins. Conserved amino acids are colored according to type: hydrophobic residues (Ala, Val, Ile, Leu, Met, Phe, and Tyr) are shown in shade of yellow and negatively charged residues (Asp, Glu) in red. The conserved motifs $(\varphi \mathrm{XX} \varphi$ or $\varphi \mathrm{XX} \varphi \varphi)$ are shown over the sequence alignment. Alpha-helical regions of p53 TAD and ACTR in the complex with NCBD are shown over the alignment and under the alignment, respectively. 
Table 1

NMR Restraints and Structural Statistics

\begin{tabular}{|c|c|c|}
\hline \multicolumn{3}{|l|}{ NMR constraints } \\
\hline & NCBD & p53 TAD \\
\hline Total NOE distance restraints & 836 & 602 \\
\hline Intra-residue $(\mathrm{i}, \mathrm{i})$ & 245 & 178 \\
\hline Sequential $(i, i+1)$ & 260 & 258 \\
\hline Medium-range $(2 \leq|\mathrm{i}-\mathrm{j}| \leq 4)$ & 247 & 154 \\
\hline Long-range $(|i-j| \geq 5)$ & 84 & 12 \\
\hline Intermolecular & \multicolumn{2}{|c|}{65} \\
\hline \multicolumn{3}{|l|}{ Dihedral angle restraints } \\
\hline$\varphi$ & 47 & 26 \\
\hline$\varphi$ & 33 & 12 \\
\hline$\chi 1$ & 7 & 7 \\
\hline \multicolumn{3}{|l|}{ Structural statistics (20 structures) } \\
\hline \multicolumn{3}{|l|}{ AMBER restraint violations } \\
\hline Maximum NOE violation $(\AA)$ & \multicolumn{2}{|c|}{0.25} \\
\hline Maximum torsion angle violation $\left({ }^{\circ}\right)$ & \multicolumn{2}{|r|}{0} \\
\hline \multicolumn{3}{|l|}{ Deviations from ideal geometry } \\
\hline Bond lengths ( & \multicolumn{2}{|c|}{$0.0102 \pm 0.0001$} \\
\hline Bond angles $\left({ }^{\circ}\right)$ & \multicolumn{2}{|c|}{$2.10 \pm 0.02$} \\
\hline \multicolumn{3}{|l|}{ AMBER energies } \\
\hline Mean restraint energy $(\mathrm{kcal} / \mathrm{mol})$ & \multicolumn{2}{|c|}{9.2} \\
\hline Mean AMBER energy (kcal/mol) & \multicolumn{2}{|c|}{-3745.9} \\
\hline \multicolumn{3}{|l|}{ RMS deviations from mean 1} \\
\hline Backbone heavy atoms $(\AA)$ & \multicolumn{2}{|c|}{0.61} \\
\hline Heavy atoms $(\AA)$ & \multicolumn{2}{|c|}{0.93} \\
\hline
\end{tabular}

PROCHECK statistics

\begin{tabular}{ll} 
Most favored region (\%) & 84.8 \\
Additionally allowed region (\%) & 14.8 \\
Generously allowed region (\%) & 0.4 \\
Disallowed region (\%) & 0.0 \\
\hline &
\end{tabular}


Table 2

Thermodynamic parameters obtained from isothermal titration calorimetry experiments for complexes of p53 TADs and NCBD

\begin{tabular}{lccc}
\hline Complex & $\Delta \mathbf{H}(\mathbf{k c a l} / \mathbf{m o l e})$ & $-\mathbf{T} \Delta \mathbf{S}(\mathbf{k c a l} / \mathbf{m o l e})$ & $\mathbf{K}_{\mathbf{d}}(\boldsymbol{\mu M})$ \\
\hline p53 (13-61)/NCBD & $-4.42 \pm 0.15$ & $-3.37 \pm 0.19$ & $3.1 \pm 0.2$ \\
p53 (13-61,D49A)/NCBD & $-2.09 \pm 0.45$ & $-5.37 \pm 0.41$ & $5.1 \pm 0.3$ \\
p53 (25-61)/NCBD & $-4.12 \pm 0.46$ & $-3.32 \pm 0.59$ & $5.4 \pm 1.8$ \\
p53 (38-61)/NCBD & $-4.60 \pm 0.12$ & $-2.26 \pm 0.10$ & $13.5 \pm 0.54$ \\
\hline
\end{tabular}

